\title{
Relationship between autophagy, apoptosis and endoplasmic reticulum stress induced by melatonin in osteoblasts by septin 7 expression
}

\author{
CUI CUI, TAO LIN, ZUNLEI GONG and YUE ZHU \\ Department of Orthopaedics, First Hospital of China Medical University, Shenyang, Liaoning 110001, P.R. China
}

Received October 30, 2018; Accepted March 17, 2020

DOI: $10.3892 / \mathrm{mmr} .2020 .11063$

\begin{abstract}
Melatonin secreted by the pineal body is associated with the occurrence and development of idiopathic scoliosis. Melatonin has a concentration-dependent dual effect on osteoblast proliferation, in which higher concentrations can inhibit osteoblast proliferation and induce apoptosis; however, the underlying mechanism remains unclear. In the present study, flow cytometry was used to demonstrate that osteoblast cells treated with melatonin exhibited significantly increased early and late stage apoptotic rates as the concentration increased. Chromatin condensation in the nucleus and apoptotic body formation could be observed using fluorescent microscopy in osteoblast cells treated with $2 \mathrm{mM}$ melatonin. Western blotting results showed that there was an upregulation in the expression of apoptosis marker proteins [poly (ADP-ribose) polymerase 1 (PARP-1)], endoplasmic reticulum stress [ERS; C/EBP homologous protein (CHOP) and glucose-regulated protein, $78 \mathrm{kDa}$ (GRP78)] and autophagy [microtubule-associated protein 1 light chain $3 \beta$ (LC3)-I/LC3II]. PARP-1 expression was not altered when treated with ERS inhibitor 4PBA and autophagy inhibitor 3MA, whereas 4PBA or 3MA in combination with $2 \mathrm{mM}$ melatonin (or the three together) significantly increased PARP-1 expression. Furthermore, the use of septin7 small interfering RNA confirmed that increased expression of GRP78 and CHOP was related to septin7, and melatoninmediated ERS was necessary for septin7 activation. These findings suggest that ERS and autophagy might occur in the early stage of treatment with a high concentration of melatonin, and each might play a protective role in promoting survival; in a later stage, ERS and autophagy might interact and contribute to the induction of apoptosis. Overall, the results indicated that septin7 may be a target protein of melatonin-induced ERS.
\end{abstract}

Correspondence to: Dr Yue Zhu or Dr Tao Lin, Department of Orthopaedics, First Hospital of China Medical University, 155 Nan Jing Bei Street, Shenyang, Liaoning 110001, P.R. China

E-mail: zhuyuedr@163.com

E-mail: taolindr@163.com

Key words: melatonin, osteoblast cells, apoptosis, endoplasmic reticulum stress, autophagy, septin7

\section{Introduction}

Idiopathic scoliosis is the most common form of scoliosis; it is characterized by a complicated three-dimensional spinal deformity that is accompanied by a rotation of the vertebrae body (1-6). Several studies have reported on the influence of the pineal body on experimentally induced spinal scoliosis, and numerous researchers hypothesize that defects in the synthesis and metabolism of melatonin secreted from the pineal body is a key factor underlying spinal scoliosis (7-9). The pineal body secretes the neuroendocrine hormone melatonin, which serves an important role in regulating physiological and pathological effects in the human body, including bone growth and vertebrae body molding $(10,11)$. A prospective study demonstrated that melatonin deficiency may play a key role in the prognosis of idiopathic scoliosis, the progress of which may be prevented by melatonin supplements (12).

Our previous studies demonstrated that melatonin exerts a dual effect on osteoblast proliferation that is dependent on concentration; $1 \mathrm{nM}-100 \mu \mathrm{M}$ melatonin can stimulate cell proliferation, whereas $1 \mathrm{mM}$ melatonin can significantly delay osteoblast proliferation (13-14). It was also found that melatonin could upregulate the concentration of calcium ions, activate the calcium pathway, disrupt homeostasis of intracellular calcium, and lead to calcium overload (15-20). Calcium overload induces endoplasmic reticulum stress (ERS), which is associated with autophagy and apoptosis. Septins belong to a class of cytoskeletal proteins with GTPase activity, which can form intracellular filamentous scaffolds. Previous studies have demonstrated that septins are involved in numerous biological processes, such as cell mitosis, polarity determination, vesicle trafficking and apoptosis (21-23). In addition, our previous study revealed that septin-7, a member of septin family, has an inhibitory effect in melatonin-induced apoptosis (15). However, the mechanisms underlying the inhibition of apoptosis by septin-7 hasn't been described comprehensively and the effect of autophagy in this process is still unclear. The present study was designed to further clarify the mechanisms by which melatonin induces osteoblast apoptosis, thus offering an improved understanding of the roles of ERS, autophagy and apoptosis in melatonin-induced osteoblasts. The insights from the present study may be useful in the investigation of melatonin as a potential treatment of idiopathic scoliosis. 


\section{Materials and methods}

Reagents. Melatonin, ERS inhibitor 4PBA, autophagy inhibitor 3MA and Hoechst 33342 stain were obtained from Sigma-Aldrich (Merck KGaA). An Annexin V-FITC/PI Apoptosis Detection kit was obtained from Nanjing KeyGen Biotech Co., Ltd. Primary antibodies against microtubule-associated protein 1 light chain $3 \beta$ (LC3; 1:1,000; cat. no. ab128025), glucose-regulated protein, $78 \mathrm{kDa}$ (GRP78; 1:1,000; cat. no. ab21685), septin7 (1:1,000; cat. no. ab186021) were obtained from Abcam. Primary antibodies against poly (ADP-ribose) polymerase 1 (PARP-1; 1:1,000; cat. no. 9532), C/EBP homologous protein (CHOP; 1:1,000; cat. no. 5554), $\beta$-actin (1:1,000; cat. no. 4970) were obtained from Cell Signaling Technology, Inc., as were fluorescent anti-rabbit secondary antibodies (1:500; cat. no. 4414).

Cell culture. The human fetal osteoblastic cell line hFOB 1.19 kindly provided by Dr Mamayannan Subramaniam (Department of Biochemistry and Molecular Biology, Mayo Clinic, Rochester, MN, USA), was maintained in a 1:1 mixture of Ham's F-12 medium/Dulbecco's modified Eagle's medium without phenol red (Gibco; Thermo Fisher Scientific, Inc.), supplemented with $10 \%$ fetal bovine serum (FBS; HyClone; GE Healthcare Life Sciences) at $37^{\circ} \mathrm{C}$ in a humidified $5 \% \mathrm{CO}_{2}$ atmosphere. The medium was changed every other day. The cells were utilized at passages $8-12$ and plated at $10^{4}$ cells $/ \mathrm{cm}^{2}$ for 4, 24 and $48 \mathrm{~h}$ before treatment. Cells were treated with melatonin, which was dissolved in $0.2 \%$ dimethyl sulfoxide (DMSO), or vehicle (0.2\% DMSO in culture medium only) media containing $10 \%$ FBS.

Apoptosis assay. Apoptosis was detected using the Annexin V-FITC/PI Apoptosis Detection kit according to the manufacturer's protocols. Briefly, hFOB 1.19 cells were seeded onto 6 -well plates $\left(5 \times 10^{5}\right.$ cells/well). After treatment with $1,2,4$ and $8 \mathrm{mM}$ melatonin for $24 \mathrm{~h}$ in $5 \% \mathrm{CO}_{2}$ at $37^{\circ} \mathrm{C}$, cells were harvested by trypsin digestion without EDTA, resuspended in $500 \mu \mathrm{l}$ final volume of binding buffer, and incubated with $5 \mu \mathrm{l}$ of Annexin V-FITC and $5 \mu \mathrm{l}$ of propidium iodide (PI) solution for $15 \mathrm{~min}$ at room temperature in the dark. Finally, cells were analyzed using a BD FACScan flow cytometer equipped with ModFit LT v3.0 (Becton, Dickinson and Company) within $1 \mathrm{~h}$. Viable cells (Annexin

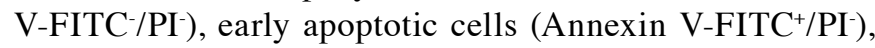
late apoptotic cells (Annexin $\mathrm{V}$-FITC ${ }^{+} / \mathrm{PI}^{+}$) and necrotic cells (Annexin V-FITC $/ \mathrm{PI}^{+}$).

Chromatin condensation assay. Chromatin condensation in the nucleus was observed using the fluorescent Hoechst 33342 stain. hFOB 1.19 were seeded onto 24 -well plates $\left(2 \times 10^{3}\right.$ cells/well). After treatment with $2 \mathrm{mM}$ melatonin for $24 \mathrm{~h}$, cells were fixed in $4 \%$ paraformaldehyde fix solution for $15 \mathrm{~min}$ at room temperature, and incubated with a concentration of $2 \mu \mathrm{g} / \mathrm{ml}$ of Hoechst 33342 for $15 \mathrm{~min}$ at room temperature in the dark. After washing three times with PBS, cells were observed and images captured under a fluorescent microscope.

Septin7 small interfering (si)RNA transfection. Cells were cultured as aforementioned. Using Lipofectamine ${ }^{\circledR} 2000$
(Invitrogen; Thermo Fisher Scientific, Inc.), according to the manufacturer's protocols, cells were transfected with $60 \mathrm{~nm}$ septin7 siRNA (forward, 5'-CGACUACAUUGAUAG UAAAUU-3' and reverse, 5'-UUUACUAUCAAUGUAGUC GAU-3') purchased from Shanghai GeneChem Co., Ltd., at $37^{\circ} \mathrm{C}$ for $48 \mathrm{~h}$. There were three control groups: i) An untreated blank control; ii) transfection reagent control (to control for potentially toxic influence of Lipofectamine 2000 and the influence of Lipofectamine 2000 on the expression of the target gene); and scramble siRNA control (5'-GAAATTTATAAC GATCAGTCT-3') purchased from Shanghai GeneChem Co., Ltd. The time interval between transfection and subsequent experimentation was $24 \mathrm{~h}$.

Overexpression plasmid construction and rescue experiment. Septin7 was cloned into GV230 plasmids (200 ng; Shanghai GeneChem Co., Ltd.) between XhoI and KpnI restriction sites to overexpress septin7 in hFOB 1.19 cells. The full-length septin7 gene $(4,377 \mathrm{bp}$; reference sequence NM_001011553) was amplified by PCR using the following primers: Forward, 5'-CTGCTCACAATAGTTGATACC CC-3' and reverse, 5'-TGTTCACTCGTGATTCTGCAT T-3'. PrimeSTAR HS DNA polymerase was obtained from GeneChem, Inc., and cycled for 30 cycles following initial denaturation $\left(98^{\circ} \mathrm{C}\right.$ for $\left.5 \mathrm{~min}\right)$ with the following parameters: $72^{\circ} \mathrm{C}$ for $8 \mathrm{~min}$. Following enzyme digestion (Exnase ${ }^{\mathrm{TM}} \mathrm{II}$; Vazyme Biotech Co., Ltd.; $1 \mu \mathrm{l}$ ) using ClonExpress II One Step Cloning kit (Vazyme Biotech Co., Ltd.) and sequencing, the PCR product was cloned into the $\mathrm{XhoI} / \mathrm{KpnI}$ sites of the GV230 expression vector. The recombinant GV230-septin7 plasmid was confirmed by endonuclease digestion and DNA sequencing (GeneChem, Inc.) prior to transfection into osteoblasts using Lipofectamine $2000\left(37^{\circ} \mathrm{C}\right.$ for $48 \mathrm{~h}$; $90 \%$ cell density) according to the manufacturer's protocols (Invitrogen; Thermo Fisher Scientific, Inc.). The rescue experiment using $45 \mathrm{nM}$ septin7 overexpression plasmid was performed following transfection of the septin7 siRNA. The time interval between transfection and subsequent experimentation was $24 \mathrm{~h}$.

Immunoblotting. Cells were lysed in RIPA buffer on ice for $30 \mathrm{~min}$. Protein fractions were centrifuged for $15 \mathrm{~min}$ at $4^{\circ} \mathrm{C}$ at $12,000 \mathrm{x} \mathrm{g}$, and supernatants containing total protein were harvested. Then, aliquots containing $50 \mu \mathrm{g}$ protein were separated by $12 \%$ SDS-PAGE and transferred onto PVDF membranes. The membranes were blocked with $5 \%$ BSA (Beyotime Institute of Biotechnology) at $4^{\circ} \mathrm{C}$ for $2 \mathrm{~h}$, and incubated with primary antibodies against PARP-1, LC3-II, GRP78, CHOP, septin7 or $\beta$-actin overnight at $4^{\circ} \mathrm{C}$. Following incubation, a fluorescent anti-rabbit secondary antibody was applied for $2 \mathrm{~h}$ at $4^{\circ} \mathrm{C}$, and blots were visualized using an ECL system (Analytik Jena US, LLC). Protein levels were normalized to the corresponding $\beta$-actin band, and the optical density was semi-quantified using ImageJ Software (National Institutes of Health).

Statistical analysis. Statistical analysis among groups was performed using one-way ANOVA with Tukey's test to evaluate the differences between melatonin, Septin 7 siRNA or overexpression plasmid groups (RNA and protein level), and two-way 
ANOVA with Tukey's test was used to evaluate the differences between melatonin, 4PBA, 3MA or combined groups (protein level). Data were expressed as the mean \pm SEM. $\mathrm{P}<0.05$ was considered to indicate a statistically significant difference.

\section{Results}

Effects of melatonin on osteoblast apoptosis. Osteoblast apoptosis was detected by flow cytometry with the Annexin V-FITC/PI Apoptosis Detection kit. The results indicated that when hFOB 1.19 cells were treated with melatonin $(1,2,4$ and $8 \mathrm{mM})$ for $24 \mathrm{~h}$, the early and late apoptotic rates of osteoblast cells increased significantly compared with the control group in a concentration-dependent manner (Fig. 1A). The inhibitory effect at $2 \mathrm{mM}$ concentration was most suitable; at 4 and $8 \mathrm{mM}$, early apoptotic rates increased significantly, resulting in cell death. Subsequently, fluorescence microscopy with the fluorescent Hoechst 33342 stain was used to observe chromatin condensation in the nucleus and apoptotic body formation. The results demonstrated that the nuclei became dense in a melatonin concentration-dependent manner (Fig. 1B).

Effects of melatonin on PARP-1 expression. The aforementioned results indicated that apoptosis rates increased in osteoblasts induced by $2 \mathrm{mM}$ melatonin. This was further examined by measuring protein expression levels of the apoptosis marker protein PARP-1 in cells treated with $2 \mathrm{mM}$ melatonin at $0,6,24$ and $48 \mathrm{~h}$ (Fig. 2). The results demonstrated that PARP-1 expression was significantly increased in a time-dependent manner.

Effects of melatonin on CHOP and GRP78 expression . CHOP and GRP78 are marker proteins of ERS-induced apoptosis; as such, the expression levels of these proteins were examined in hFOB 1.19 cells treated with $2 \mathrm{mM}$ melatonin for $0,6,24$ or $48 \mathrm{~h}$. Western blotting results indicated that CHOP and GRP78 expressions increased significantly over time compared with treatment for $0 \mathrm{~h}$, and this was in a time-dependent manner (Fig. 3A and B, respectively).

Effects of melatonin on osteoblast autophagy. To examine the role of autophagy in melatonin-induced osteoblast apoptosis, expression levels of the autophagy marker protein LC3-I/II was examined in cells treated with $2 \mathrm{mM}$ melatonin at 6,24 and $48 \mathrm{~h}$. As presented in Fig. 4, LC3-II protein expression increased significantly over time compared with the control $(\mathrm{P}<0.05)$, with the highest levels observed at $48 \mathrm{~h}$.

Relationship between apoptosis, autophagy and ERS. To further investigate the association between apoptosis, autophagy and ERS in melatonin-treated osteoblasts, cells were incubated with either $2 \mathrm{mM}$ melatonin, 4PBA (ERS inhibitor), 3MA (autophagy inhibitor), or two or three of these in combination for $24 \mathrm{~h}$, and the expression levels of PARP-1 protein was determined using western blotting. The results showed that expression of PARP-1 was not significantly different when cells were treated with 4PBA or 3MA alone compared with the control; however, when osteoblast cells were treated with a combination of $4 \mathrm{PBA}$ or $3 \mathrm{MA}$ and $2 \mathrm{mM}$ melatonin (or all three) for $24 \mathrm{~h}$, the expression of PARP-1 increased significantly compared with the control (Fig. 5).

Relationship between septin7 and melatonin-induced ERS. To improve the understanding of the relationship between septin7 and ERS in melatonin-induced osteoblasts, septin7 expression was knocked down using siRNA. Osteoblasts transfected with septin7 siRNA exhibited a significant decrease in septin7 mRNA expression compared with the scramble siRNA, confirming successful transfection (Fig. 6A). septin7 siRNA transfection led to a decrease in GRP78 and CHOP protein expression levels (Fig. 6B), although the changes were not significant, even when in combination with melatonin. These data suggested that melatonin-mediated ERS may induce osteoblast proliferation by regulating septin7 expression.

It was also determined whether exogenous expression of septin7 could rescue the inhibition of GRP78 and CHOP expression caused by septin7 knockdown in osteoblasts by co-transfections with a septin7 overexpression plasmid. The expression levels of septin7, GRP78 and CHOP proteins were evaluated by western blotting. As shown in Fig. 6B, exogenous expression of septin7 restored cellular septin7 levels in septin7 knockdown cells induced by melatonin. It was also found that when transfected with the septin7 overexpression plasmid, osteoblasts showed significantly higher GRP78 and CHOP expression compared with the control.

\section{Discussion}

Melatonin is a neuroendocrine hormone mainly secreted by the pineal body that plays an important part in regulating physiological and pathological processes in humans, such as bone growth and vertebral body molding (24-28). Melatonin has been shown to trigger apoptosis in human alveolar rhabdomyosarcoma cells and could be considered a promising drug for future multitargeted therapies (28), but the role of melatonin in osteoblast apoptosis remains unclear. Our previous study demonstrated that a high concentration of melatonin could increase the concentration of calcium ions, causing an imbalance in the homeostasis of intracellular calcium, thus leading to calcium overload (15-20). Calcium overload induces ERS, which is associated with autophagy and apoptosis. But in our previous studies, the role of autophagy in melatonin-induced osteoblast apoptosis and the relationship between autophagy and ERS were not investigated, thus leading us to perform the present study to explore this. Based on preliminary experiments, four melatonin concentrations were chosen as they have been shown to have negative effects in vitro: $1,2,4$ and $8 \mathrm{mM}$. In this work, it was confirmed that $2 \mathrm{mM}$ melatonin readily induced apoptosis. In addition, a high concentration of melatonin caused intracellular calcium overload, which resulted in ERS, induced osteoblast autophagy and promoted osteoblast apoptosis.

Apoptosis is a type of programmed cell death controlled by genes, including $\mathrm{Bax}$ and Bcl-2, that occurs as a homeostatic mechanism to maintain cell populations in the internal environment $(11,29)$. Tumor cell studies have shown that melatonin can induce apoptosis (30); the present study demonstrated that early and late apoptotic rates of osteoblast 
A
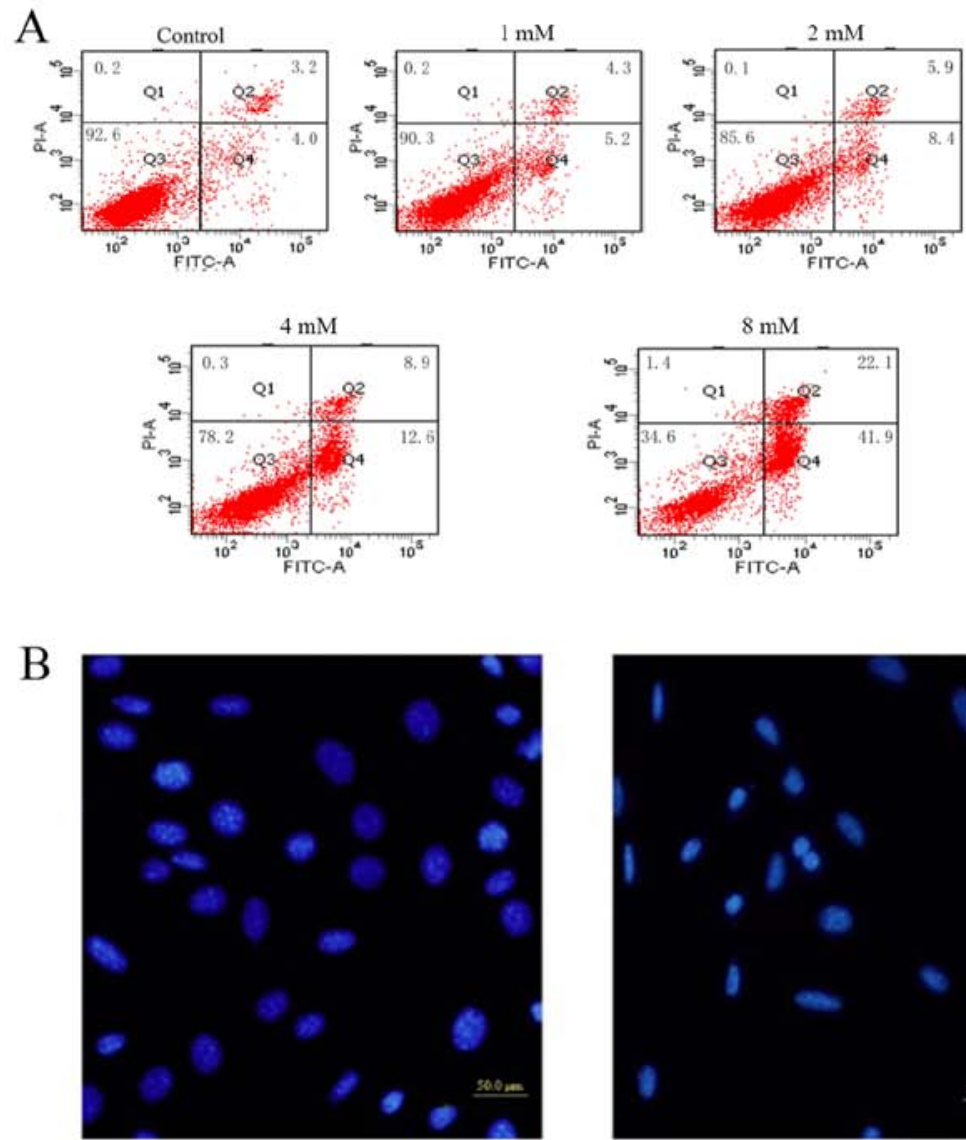

$1 \mathrm{mM}$

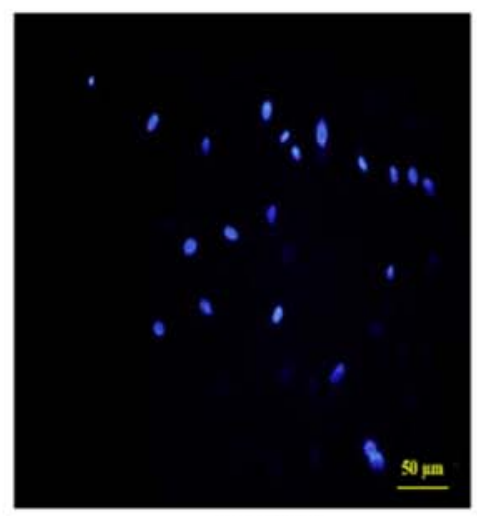

$4 \mathrm{mM}$
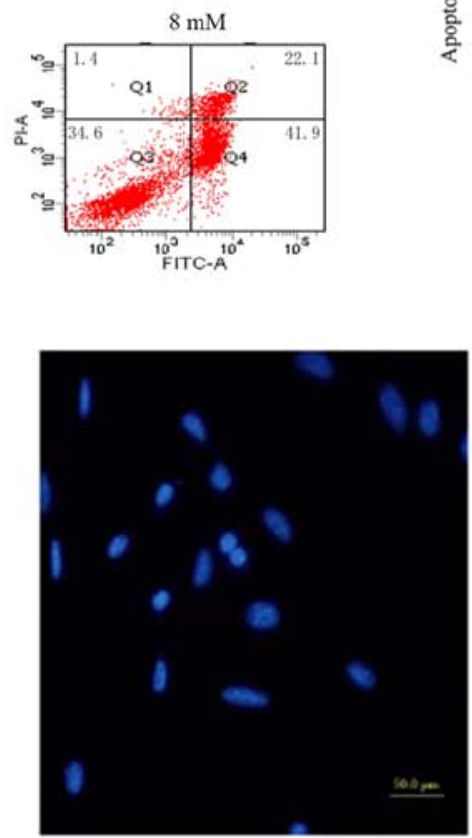

$2 \mathrm{mM}$

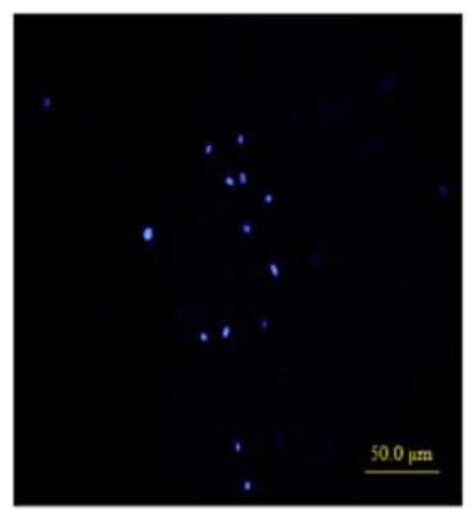

$8 \mathrm{mM}$

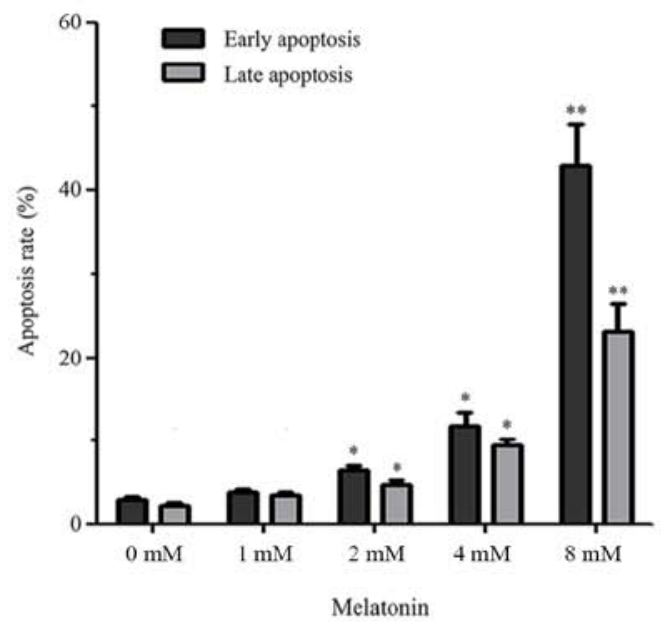

Figure 1. Effects of melatonin on apoptosis in hFOB 1.19 osteoblast cells. (A) Apoptotic rates of hFOB 1.19 cells treated with melatonin at various concentrations (1, 2, 4 and $8 \mathrm{mM})$ were determined using an Annexin V-FITC/PI kit for $24 \mathrm{~h}$. (B) Chromatin condensation in the nucleus were observed in melatonin-induced osteoblast cells $(1,2,4$ and $8 \mathrm{mM})$ using Hoechst 33342 stain with a fluorescent microscope. Experiments were performed in triplicate, and data are shown as the mean \pm SEM. ${ }^{*} \mathrm{P}<0.05,{ }^{* *} \mathrm{P}<0.01$ vs. Control. PI, propidium iodide.

cells treated with melatonin increased significantly as the concentration increased. The data also indicated that chromatin condensation in the nucleus and high expression of PARP-1 could be observed in osteoblast cells treated with $2 \mathrm{mM}$ melatonin. PARP-1 is an apoptosis marker protein and is closely related to the repair of DNA under stress (31); it is activated by recognizing the DNA fragment of structural damage and is considered to be the receptor of DNA damage (32). The findings of the present study indicate that melatonin may induce osteoblast apoptosis, although the mechanism is unclear.
ERS is an essential biological phenomenon that can be activated by a number of conditions, including fatigue, calcium imbalance and oxidative stress (33). The endoplasmic reticulum activates a series of unfolded protein responses to protect cells from damage and promote cell survival. However, when the ERS is too strong or has endured too much damage to repair, the endoplasmic reticulum can initiate a GRP78 or CHOP pathway to induce apoptosis (19). Previous experiments by the authors demonstrated that a high concentration of melatonin can cause calcium overload and inhibit osteoblast cell proliferation (13-15). Other research has demonstrated 

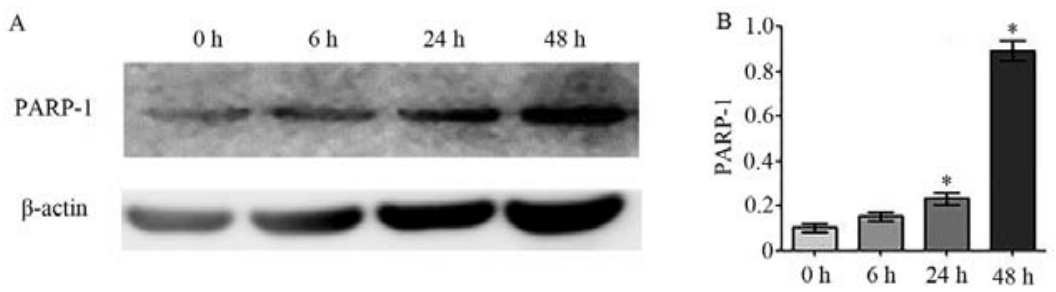

Figure 2. Effects of melatonin on PARP-1 expression in hFOB 1.19 osteoblast cells. (A) Representative western blotting images showing PARP-1 protein expression levels in $\mathrm{hFOB} 1.19$ cells treated with $2 \mathrm{mM}$ concentration of melatonin for $0,6,24$ and $48 \mathrm{~h}$. (B) Corresponding densitometric analysis of the data presented in (A) $\beta$-actin was used as a loading control. Experiments were performed in triplicate, and data are shown as the mean $\pm \mathrm{SEM}$. ${ }^{*} \mathrm{P}<0.05,{ }^{* *} \mathrm{P}<0.01$ vs. 0 h. PARP-1, poly (ADP-ribose) polymerase 1.

A

$\mathrm{C}$

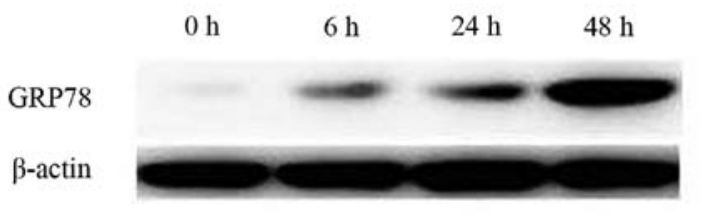

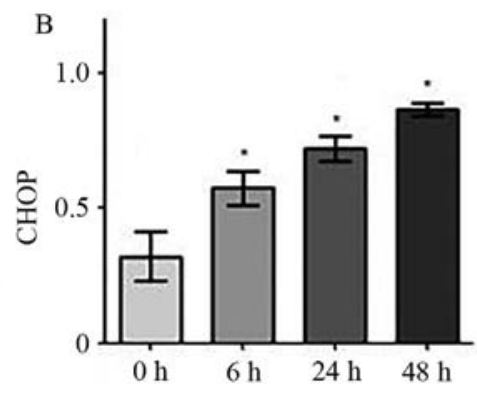

D

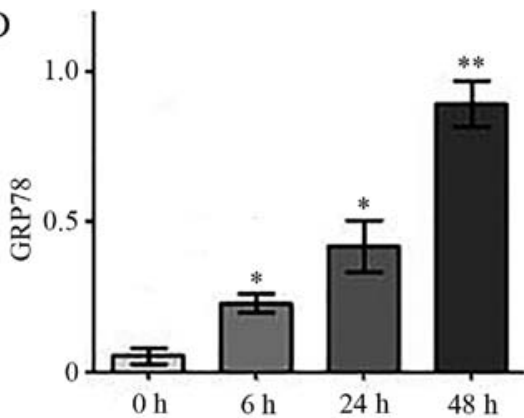

Figure 3. Effects of melatonin on CHOP and GRP78 expression levels in hFOB 1.19 osteoblast cells. (A) Representative western blotting image showing CHOP protein expression. (B) Corresponding densitometric analysis of the data presented in (A). (C) Representative western blotting image showing GRP78 protein levels. (D) Corresponding densitometric analysis of the data presented in (C) $\beta$-actin was used as a loading control. Experiments were performed in triplicate, and data are shown as the mean \pm SEM. ${ }^{*} \mathrm{P}<0.05,{ }^{* *} \mathrm{P}<0.01$ vs. 0 h. CHOP, C/EBP homologous protein; GRP78, glucose-regulated protein, $78 \mathrm{kDa}$.
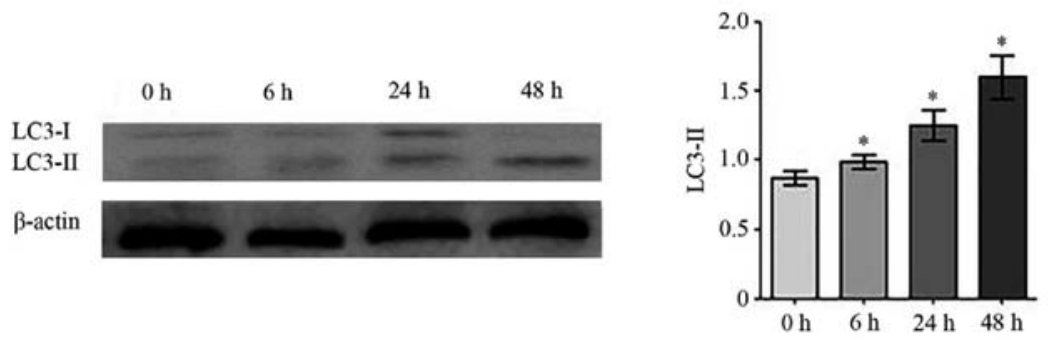

Figure 4. Effects of melatonin on osteoblast autophagy in hFOB 1.19 cells. The effect of melatonin $(2 \mathrm{mM})$ on LC3-II protein expression levels was measured by western blotting and densitometric analysis. $\beta$-actin was used as a loading control. Experiments were performed in triplicate, and data are shown as the mean \pm SEM. ${ }^{*} \mathrm{P}<0.05$ vs. 0 h. LC3, microtubule-associated protein 1 light chain $3 \beta$.

that melatonin modulates ERS and the AKT/glycogen synthase kinase- $3 \beta$ signaling pathway in a rat model of renal warm ischemia reperfusion (34); thus, we hypothesized that melatonin-induced osteoblast apoptosis is related to ERS. To examine this possibility, the effect of melatonin on GRP78 and CHOP expression was investigated in the present study. The results showed that melatonin increased GRP78 and CHOP expression as treatment time increased, which suggested that melatonin activated the ERS to initiate a specific apoptotic pathway to induce apoptosis (19).

Conversely, activated unfolded protein responses can initiate the autophagy pathway to eliminate the misfolded protein and restore homeostasis of the endoplasmic reticulum to promote cell survival; however, an excessive autophagy pathway can also cause programmed cell death (35). A previous study also examined the involvement of autophagy in melatonin-induced 
A

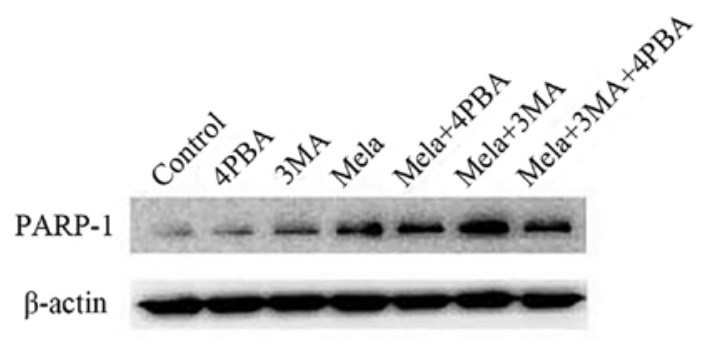

B

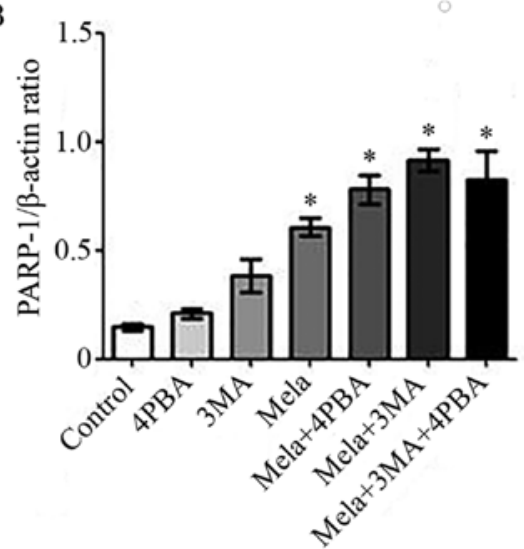

Figure 5. Relationship between apoptosis, autophagy and ERS in hFOB 1.19 cells. Osteoblasts were treated with 4PBA (an ERS inhibitor), 3MA (an autophagy inhibitor) or $2 \mathrm{mM}$ melatonin alone or in various combinations for $24 \mathrm{~h}$. (A) Representative western blotting image showing PARP-1 expression. (B) Corresponding densitometric analysis of the data presented in (A). Experiments were performed in triplicate, and data are shown as the mean \pm SEM. "P<0.05 vs. control. ERS, endoplasmic reticulum stress; mela, melatonin; PARP-1, poly (ADP-ribose) polymerase 1.
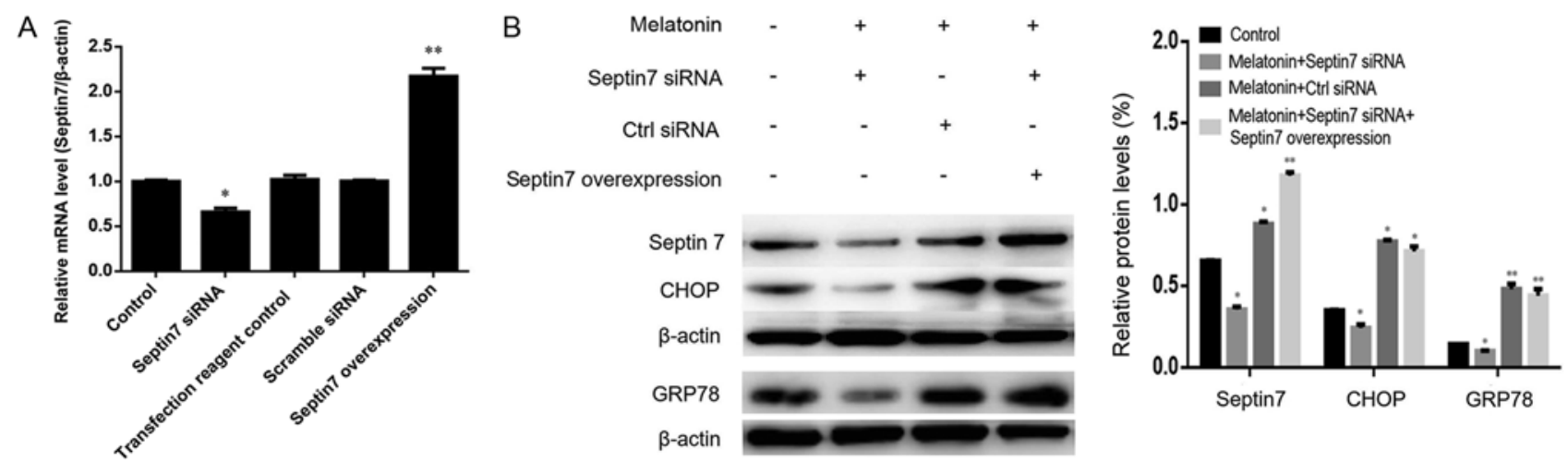

Figure 6. Relationship between septin7 and melatonin-induced endoplasmic reticulum stress in hFOB 1.19 cells. (A) Septin7 mRNA expression detected by reverse transcription-quantitative PCR in osteoblasts transfected with septin7 siRNA or overexpression vector, and corresponding controls. (B) Osteoblasts were treated for $24 \mathrm{~h}$ with $2 \mathrm{mM}$ melatonin, septin7 siRNA or septin7 overexpression RNA alone or combined (the group 4 cells were transfected with si-septin7, and subsequently transfected with septin7 overexpression vector.). Protein expression levels were analyzed by western blotting. Experiments were performed in triplicate, and data are shown as the mean \pm SEM. ${ }^{*} \mathrm{P}<0.05$ vs. scramble siRNA; ${ }^{* *} \mathrm{P}<0.01$ vs. empty vector control.

osteoblasts (36). Autophagy is an important cellular mechanism that degrades damaged macromolecules and cytoplasmic organelles; it plays a key role in cell survival, renewal, substance reuse and maintenance of internal homeostasis (37-39). The role of autophagy in bone metabolism has been previously reported. For example, autophagy attenuates oxidative stress-induced apoptosis of MC3T3-E1 osteoblasts (40). Qi et al (41) indicated that autophagy maintains the function of bone marrow mesenchymal stem cells to prevent estrogen deficiency-induced osteoporosis. The present findings showed that autophagy was triggered when osteoblasts were treated with melatonin, and the degree of autophagy increased gradually over time, as shown by the autophagy marker LC3-II. Apoptosis and autophagy are two programmed cell death processes that operate independently in numerous biological activities, this includes roles in morphology, as biochemical indicators and as regulators of cell death (42-46). To further understand the relationship between ERS, autophagy and apoptosis in melatonin-induced osteoblasts, osteoblasts were treated with either the ERS inhibitor 4PBA, autophagy inhibitor 3MA and $2 \mathrm{mM}$ melatonin, or all three, after which PARP-1 expression increased significantly, indicating an increase in apoptosis. However in the present study, 4PBA and 3MA could expand the effect of melatonin, suggesting that both ERS and autophagy can play a protective role in promoting survival, which attenuated melatonin-induced apoptosis to some extent.

The endoplasmic reticulum is sensitive to stress. It maintains its structure and function by regulating membrane proteins and selectively transports these proteins to transmit biological effects (47). It is currently unclear how the endoplasmic reticulum is involved in the melatonin-induced changes in protein expression and how it transports the related membrane protein to transmit the biological information to the cell membrane. A previous study by the authors found that melatonin treatment induced osteoblast proliferation, and this was associated with septin7 protein expression (15). Another previous study has demonstrated that septin4 is highly expressed on the endoplasmic reticulum and Golgi membrane of HeLa cells, as detected using the siRNA screen technique (48). When stimulated, septin4 redistributes rapidly on the plasma membrane, resulting in an influx of calcium ions in the cell membrane, suggesting that the septin superprotein 
family have roles in apoptosis via the induction of ERS. The septin7 superprotein family may also be the target protein of melatonin acting on osteoblasts, which can initiate the upregulation of calcium ions and cause calcium overload. The findings of the present study indicated that septin 7 may be a target protein of melatonin-induced ERS. Melatonin acting on septin7 at the endoplasmic reticulum membrane may initiate ERS and induce osteoblast autophagy followed by osteoblast apoptosis as autophagy increases. However, when septin7 was knocked down and overexpressed the results were not significant, which suggests that there may be another target protein involved.

In conclusion, results from the present study suggested that ERS and autophagy may occur in an early state of treatment with a high concentration of melatonin ( $\geq 2 \mathrm{mM})$, both of which can play a protective role in promoting survival. In later stages of treatment $(>48 \mathrm{~h}$ ), they might interact and contribute to the induction of apoptosis. Moreover, septin7 may be a target protein of melatonin-induced ERS. These data provide a theoretical basis and new ideas for therapies using melatonin to treat patients with idiopathic scoliosis.

\section{Acknowledgements}

The authors would like to acknowledge Dr M. Subramaniam (The Department of Biochemistry and Molecular Biology Research, Mayo Clinic, Rochester, MN, USA) for providing the hFOB 1.19 cell line.

\section{Funding}

This study was supported by two grants from The National Natural Foundation Science of China (grant nos. 81472044 and 81271939) and a grant from Shenyang Science and Technology Foundation of Population and Health Project (grant no. 17230904).

\section{Availability of data and materials}

All data generated or analyzed during this study are included in this published article.

\section{Authors' contributions}

CC contributed to the study design, data collection and analysis, as well as the writing of the manuscript. YZ conceived, designed and coordinated the study. TL and ZG contributed to the experimental methods. All authors read and approved the final manuscript.

\section{Ethics approval and consent to patriciate}

Not applicable.

\section{Patient consent for publication}

Not applicable.

\section{Competing interests}

The authors declare that they have no competing interests.

\section{References}

1. Burwell RG, Dangerfield PH, Moulton A and Anderson SI: Etiologic theories of idiopathic scoliosis: Autonomic nervous system and the leptin-sympathetic nervous system concept for the pathogenesis of adolescent idiopathic scoliosis. Stud Health Technol Inform 140: 197-207, 2008

2. Letellier K, Azeddine B, Blain S, Turgeon I, Wang da S, Boiro MS, Moldovan F, Labelle H, Poitras B, Rivard CH, et al: Etiopathogenesis of adolescent idiopathic scoliosis and new molecular concepts. Med Sci (Paris) 23: 910-916, 2007 (In French)

3. Carpintero P, Mesa M, Garcia J and Carpintero A: Scoliosis induced by asymmetric lordosis and rotation: An experimental study. Spine (Phila Pa 1976) 22: 2202-2206, 1997.

4. Azeddine B, Letellier K, Wang da S, Moldovan F and Moreau A: Molecular determinants of melatonin signaling dysfunction in adolescent idiopathic scoliosis. Clin Orthop Relat Res 462: 45-52, 2007.

5. Grivas TB, Vasiliadis E, Mouzakis V, Mihas C and Koufopoulos G: Association between adolescent idiopathic scoliosis prevalence and age at menarche in different geographic latitudes. Scoliosis 23: 1-9, 2006.

6. Weinstein SL and Dolan LA: The evidence base for the prognosis and treatment of adolescent idiopathic scoliosis: The 2015 orthopaedic research and education foundation clinical research award. J Bone Joint Surg Am 97: 1899-1903, 2015.

7. Poon AM, Cheung KM, Lu DS and Leong JC: Changes in melatonin receptors in relation to the development of scoliosis in pinealectomized chickens. Spine (Phila Pa 1976) 31: 2043-2047, 2006.

8. Machida M, Dubousset J, Yamada T, Kimura J, Saito M, Shiraishi T and Yamagishi M: Experimental scoliosis in melatonin-deficient C57BL/6J mice without pinealectomy. J Pineal Res 41: 1-7, 2006.

9. Cheung KM, Wang T, Poon AM, Carl A, Tranmer B, Hu Y, Luk KD and Leong JC: The effect of pinealectomy on scoliosis development in young nonhuman primates. Spine (Phila Pa 1976) 30: 2009-2013, 2005.

10. Maria S and Witt-Enderby PA: Melatonin effects on bone: Potential use for the prevention and treatment for osteopenia, osteoporosis, and periodontal disease and for use in bone-grafting procedures. J Pineal Res 56: 115-125, 2014.

11. Proksch S, Strobel SL, Vach K, Abouassi T, Tomakidi P, Ratka-Krüger P and Hellwig E: Melatonin as a candidate therapeutic drug for protecting bone cells from chlorhexidine-induced damage. J Periodontol 85: e379-e389, 2014.

12. Goultidis TT, Papavasiliou KA, Petropoulos AS, Philippopoulos A and Kapetanos GA: Higher levels of melatonin in early stages of adolescent idiopathic scoliosis: Toward a new scenario. J Pediatr Orthop 34: 768-773, 2014.

13. Liu L, Zhu Y, Xu Y and Reiter RJ: Prevention of ERK activation involves melatonin-induced $\mathrm{G}(1)$ and $\mathrm{G}(2) / \mathrm{M}$ phase arrest in the human osteoblastic cell line hFOB 1.19. J Pineal Res 53: 60-66, 2012.

14. Liu L, Zhu Y, Xu Y and Reiter RJ: Melatonin delays cell proliferation by inducing $\mathrm{G} 1$ and $\mathrm{G} 2 / \mathrm{M}$ phase arrest in a human osteoblastic cell line hFOB 1.19. J Pineal Res 50: 222-231, 2011.

15. Meng X, Zhu Y, Tao L, Zhao S and Qiu S: Overexpression of septin-7 inhibits melatonin-induced cell apoptosis in human fetal osteoblastic cells via suppression of endoplasmic reticulum stress. Mol Med Rep 17: 4817-4822, 2018

16. Tao $\mathrm{L}$ and Zhu Y: Melatonin regulates CRE-dependent gene transcription underlying osteoblast proliferation by activating Src and PKA in parallel. Am J Transl Res 10: 86-100, 2018.

17. Liu L, Zhu Y, Han X and Wu Y: The creation of scoliosis by scapula-to-contralateral ilium tethering procedure in bipedal rats a kyphoscoliosis model. Spine (Phila Pa 1976) 6: 1340-1349, 2011.

18. Xiong XC, Zhu Y, Ge R, Liu LF and Yuan W: Effect of melatonin on the extracellular-regulated kinase signal pathway activation and human osteoblastic cell line hFOB 1.19 proliferation. Int J Mol Sci 16: 10337-10353, 2015.

19. Meng X, Zhu Y, Tao L, Zhao S and Qiu S: Periostin has a protective role in melatonin-induced cell apoptosis by inhibiting the eIF2 $\alpha$-ATF4 pathway in human osteoblasts. Int J Mol Med 41: 1003-1012, 2018.

20. Meng X, Zhu Y, Tao L, Zhao S and Qiu S: MicroRNA-125b-1-3p mediates intervertebral disc degeneration in rats by targeting teashirt zinc finger homeobox 3. Exp Ther Med 15: 2627-2633, 2018. 
21. He X, Bao J, Chen J, Sun X, Wang J, Zhu D, Song K, Peng W, $\mathrm{Xu} \mathrm{T}$ and Duan Y: Adenovirus-mediated over-expression of Septin4 ameliorates hepatic fibrosis in mouse livers infected with Schistosoma japonicum. Parasitol Int 64: 487-492, 2015.

22. Shen S, Liu M, Wu Y, Saiyin H, Liu G and Yu L: Involvement of SEPT4_i1 in hepatocellular carcinoma: SEPT4_i1 regulates susceptibility to apoptosis in hepatocellular carcinoma cells. Mol Biol Rep 39: 4519-4526, 2012.

23. Kinoshita M: Diversity of septin scaffolds. Curr Opin Cell Biol 18: 54-60, 2006.

24. Han Y, Kim YM, Kim HS and Lee KY: Melatonin promotes osteoblast differentiation by regulating Osterix protein stability and expression. Sci Rep 7: 5716, 2017.

25. Dimitri P and Rosen C: The central nervous system and bone metabolism: An evolving story. Calcif Tissue Int 100: 476-485, 2017

26. Vriend $\mathrm{J}$ and Reiter RJ: Melatonin, bone regulation and the ubiquitin-proteasome connection: A review. Life Sci 145: 152-160, 2016

27. Roforth MM, Farr JN, Fujita K, McCready LK, Atkinson EJ, Therneau TM, Cunningham JM, Drake MT, Monroe DG and Khosla S: Global transcriptional profiling using RNA sequencing and DNA methylation patterns in highly enriched mesenchymal cells from young versus elderly women. Bone 76: 49-57, 2015.

28. Burattini S, Battistelli M, Codenotti S, Falcieri E, Fanzani A and Salucci S: Melatonin action in tumor skeletal muscle cells: An ultrastructural study. Acta Histochem 118: 278-85, 2016.

29. Maiuri MC, Zalckvar E, Kimchi A and Kroemer G: Self-eating and self-killing: Crosstalk between autophagy and apoptosis. Nat Rev Mol Cell Biol 8: 741-752, 2007.

30. Pourhanifeh MH, Sharifi M, Reiter RJ, Davoodabadi A and Asemi Z: Melatonin and non-small cell lung cancer: New insights into signaling pathways. Cancer Cell Int 19: 131, 2019.

31. Singh MP, Chauhan AK and Kang SC: Morin hydrate ameliorates cisplatin-induced ER stress, inflammation and autophagy in HEK-293 cells and mice kidney via PARP-1 regulation. Int Immunopharmacol 56: 156-167, 2018.

32. Wang Y, Luo W and Wang Y: PARP-1 and its associated nucleases in DNA damage response. DNA Repair (Amst) 81: 102651, 2019.

33. Hengartner MO: The biochemistry of apoptosis. Nature 407 $770-776,2000$

34. Hadj Ayed Tka K, Mahfoudh Boussaid A, Zaouali MA Kammoun R, Bejaoui M, Ghoul Mazgar S, Rosello Catafau J and Ben Abdennebi H: Melatonin modulates endoplasmic reticulum stress and Akt/GSK3-beta signaling pathway in a rat model of renal warm ischemia reperfusion. Anal Cell Pathol (Amst) 2015: 635172, 2015.

35. Gump JM and Thorburn A: Autophagy and apoptosis: What is the connection? Trends Cell Biol 21: 387-392, 2011.

36. Zhang WL, Meng HZ, Yang RF, Yang MW, Sun GH, Liu JH, Shi PX, Liu F and Yang B: Melatonin suppresses autophagy in type 2 diabetic osteoporosis. Oncotarget 7: 52179-52194, 2016.

37. Sun X, Yang X, Zhao Y, Li Y and Guo L: Effects of 17ß-estradiol on mitophagy in the murine MC3T3-E1 osteoblast cell line is mediated via $\mathrm{G}$ protein-coupled estrogen receptor and the ERK1/2 signaling pathway. Med Sci Monit 24: 903-911, 2018.
38. Liu W, Zhao Z, Na Y, Meng C, Wang J and Bai R Dexamethasone-induced production of reactive oxygen species promotes apoptosis via endoplasmic reticulum stress and autophagy in MC3T3-E1 cells. Int J Mol Med 41: 2028-2036, 2018.

39. Li X, Lu Q, Xie W, Wang Y and Wang G: Anti-tumor effects of triptolide on angiogenesis and cell apoptosis in osteosarcoma cells by inducing autophagy via repressing Wnt/ $\beta$-Catenin signaling. Biochem Biophys Res Commun 496: 443-449, 2018.

40. Yoo YM, Han TY and Kim HS: Melatonin suppresses autophagy induced by clinostat in preosteoblast MC3T3-E1 cells. Int J Mol Sci 17: 526, 2016.

41. Qi M, Zhang L, Ma Y, Shuai Y, Li L, Luo K, Liu W and Jin Y: Autophagy maintains the function of bone marrow mesenchymal stem cells to prevent estrogen deficiency-induced osteoporosis. Theranostics 7: 4498-4516, 2017.

42. Song HS, Jang S and Kang SC: Bavachalcone from Cullen corylifolium induces apoptosis and autophagy in HepG2 cells. Phytomedicine 40: 37-47, 2018

43. San-Miguel B, Crespo I, Sánchez DI, González-Fernández B, Ortiz de Urbina JJ, Tuñón MJ and González-Gallego J: Melatonin inhibits autophagy and endoplasmic reticulum stress in mice with carbon tetrachloride-induced fibrosis. J Pineal Res 59: 151-162, 2015.

44. De Luxán-Delgado B, Potes Y, Rubio-González A, Caballero B, Solano JJ, Fernández-Fernández M, Bermúdez M, Rodrigues Moreira Guimarães M, Vega-Naredo I, Boga JA and Coto-Montes A: Melatonin reduces endoplasmic reticulum stress and autophagy in liver of leptin-deficient mice. J Pineal Res 61: $108-123,2016$.

45. Moreira AJ, Ordoñez R, Cerski CT, Picada JN, García-Palomo A, Marroni NP, Mauriz JL and González-Gallego J: Melatonin activates endoplasmic reticulum stress and apoptosis in rats with diethylnitrosamine-induced hepatocarcinogenesis. PLoS One 10: e0144517, 2015.

46. Wongprayoon $\mathrm{P}$ and Govitrapong $\mathrm{P}$ : Melatonin protects SH-SY5Y neuronal cells against methamphetamine-induced endoplasmic reticulum stress and apoptotic cell death. Neurotox Res 31: 1-10, 2017.

47. Ron D and Walter P: Signal integration in the endoplasmic reticulum unfolded protein response. Nat Rev Mol Cell Biol 8: 519-529, 2007.

48. Sharma S, Quintana A, Findlay GM, Mettlen M, Baust B, Jain M Nilsson R, Rao A and Hogan PG: An siRNA screen for NFAT activation identifies septins as coordinators of store-operated $\mathrm{Ca}^{2+}$ entry. Nature 499: 238-242, 2013.

This work is licensed under a Creative Commons Attribution-NonCommercial-NoDerivatives 4.0 International (CC BY-NC-ND 4.0) License. 